\title{
Balloon dilatation of the aortic valve for congenital aortic stenosis in childhood
}

I D SULLIVAN, C WREN, $\dagger$ H BAIN, $\dagger$ S HUNTER, $\dagger$ P G REES, * J F N TAYLOR, * C BULL, J E DEANFIELD*

From the ${ }^{\star}$ Hospital for Sick Children, Great Ormond Street, London and †Freeman Hospital, Newcastle upon Tyne

SUMMARY Balloon dilatation of the aortic valve was attempted in 34 consecutive children aged 16 months to 17 years (median 7 years), weight range 9-60 (median 22) kg. Previous surgica $\overline{\$ 0}$ valvotomy had been performed in two patients (twice in one of them). The valve was not crossed ing one patient. In the remaining 33 patients the pressure difference between the left ventricle and the ascending aorta during systole was reduced from 71 (30) to 28 (19) $\mathrm{mm} \mathrm{Hg}$. In 24 patient recatheterisation 2-19 (mean 9) months later showed gradients that were similar to thos immediately after balloon dilatation $(35(20) v 31(20) \mathrm{mm} \mathrm{Hg}$ ). The two patients with the highes residual gradients immediately after balloon dilatation showed a spontaneous reduction in gradient at repeat catheterisation, whereas the patient who had twice had previous surgical valvotom 6 showed an increase in gradient from 37 to $99 \mathrm{~mm} \mathrm{Hg}$ over nine months and required aortic root replacement. Balloon dilatation was repeated in two patients and this caused a further reduction io gradient. New aortic regurgitation occurred in nine $(27 \%)$ patients (grade I, 8; grade II, 1) an

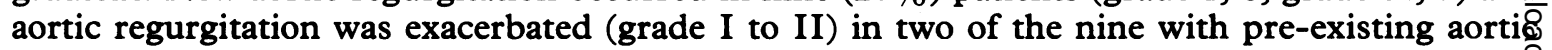
regurgitation. External iliac artery avulsion occurred in one $(3 \%)$ patient and two $(6 \%)$ require $\$$ intravenous streptokinase because the femoral artery became occluded. There were no other complications. Open valvotomy was performed in the child in whom the valve was not crossed, bu no other child required aortic valve operation.

Balloon dilatation of the aortic valve gave reasonable short term palliation and was well tolerated? It is an alternative to surgical valvotomy for initial palliation of congenital aortic stenosis in man children.

Relief of obstruction in congenital stenosis of the aortic valve in childhood has usually been by open surgical valvotomy. This, however, is a palliative technique, and survivors are at risk of late death and infective endocarditis and may need reoperation..$^{1-3}$ Aortic valve replacement in childhood is inappropriate because the patient will continue to grow. Also bioprosthetic valves are subject to calcification, and patients with mechanical prostheses will need anticoagulation. So a less hazardous non-surgical palliative technique to relieve aortic stenosis would have major clinical advantages.

Our success with balloon dilatation for pulmonary

Requests for reprints to Dr I D Sullivan, Cardiothoracic Unit, Hospital for Sick Children, Great Ormond Street, London WC1N $3 \mathrm{JH}$.

Accepted for publication 12 October 1988 stenosis, ${ }^{4}$ together with early reports of successfuB balloon dilatation in both congenital ${ }^{56}$ and, later acquired aortic valve stenosis, ${ }^{7}$ led us to extend the technique to a consecutive series of infants and children with congenital aortic stenosis. We have. already reported our experience with balloon dilata tion of the aortic valve in neonates and infants ${ }^{8}-a{ }^{8}$ age group with problems that differ from those of older children with aortic stenosis. Our results in the smallest and sickest neonates were disappointing an similar to those associated with valvotomy in these patients. In the present study we report our earl experience with balloon dilatation of the aortic valve for congenital aortic stenosis in childhood.

\section{Patients and methods}

Balloon dilatation of the aortic valve was attempted iv 
34 consecutive children with aortic stenosis up to March 1988 at The Hospital for Sick Children, London (21 patients since April 1986) and Freeman Hospital, Newcastle upon Tyne (13 patients since June 1985). Patients were aged from 16 months to 17 years (median 7 years) and weighed 9-60 (median 22) $\mathrm{kg}$.

Indications for balloon dilatation of the aortic valve were clinical evidence of at least moderate stenosis, ${ }^{1}$ together with a measured peak to peak systolic pressure difference at previous catheterisation or Doppler peak instantaneous gradient of $>50 \mathrm{~mm} \mathrm{Hg}$. Most were symptom free. Only one child had heart failure and only two of the remainder reported symptoms. Previous surgical valvotomy had been performed in two $(6 \%)$ patients (on two occasions in one of them). Previous coarctation repair had been performed in four (13\%) patients, with balloon dilatation of recoarctation in one. None had a residual coarctation gradient of $>10 \mathrm{~mm} \mathrm{Hg}$.

Cross sectional echocardiographic measurement of the diameter of the aortic root was performed at the level of attachment of the aortic valve cusps in the parasternal long axis plane. Cardiac catheterisation was performed by standard techniques. General anaesthesia was used in $31(91 \%)$ patients and local anaesthesia and basal sedation in three $(9 \%)$. The femoral artery was entered via percutaneous puncture. A catheter to monitor arterial pressure was placed in the ascending aorta via the contralateral femoral artery in 24 patients to allow simultaneous measurement of pressure in the left ventricle and ascending aorta. In the remainder the transvalvar aortic pressure difference was measured during systole by pull back from the left ventricle to the ascending aorta. These patients were usually those of smaller body weight and the procedure avoided a second arterial puncture. Heparin (100 units/kg) was given. Angiography of the ascending aorta was used to assess the grade of aortic regurgitation and also to confirm the diameter of the aortic valve. This was estimated from the known catheter dimension.

Aortic regurgitation was graded as follows: (I) "whiff' of regurgitated contrast without outlining of the left ventricular cavity; (II) left ventricular cavity outlined by contrast which was always of lower density than that in the ascending aorta; (III) left ventricular cavity outlined by contrast medium which was of equal density to that in the ascending aorta in more than three beats; (IV) equalisation of contrast density in the left ventricle and ascending aorta within three beats.

The left ventricle was entered retrogradely with an end hole catheter, usually over a guide wire. An exchange guide wire was introduced to the left ventricle via this catheter. The soft tip of the wire was curled in the left ventricular cavity. The balloon catheter (Meditech, Surgimed, or Cook) was introduced directly over the wire without an arterial sheath. A catheter with an inflated balloon size at least $1 \mathrm{~mm}$ less than the measured aortic root diameter was selected. The balloon was usually 3 or $4 \mathrm{~cm}$ long, although we used longer balloons $(5.5$ or $8 \mathrm{~cm}$ ) in large patients in the latter part of the study. The balloon was purged of air, positioned across the aortic valve, and then inflated $2-5$ times by hand with dilute contrast medium, the aim being to abolish the "waist" in the partly inflated balloon. Two balloons, each of $15 \mathrm{~mm}$ diameter, were inflated simultaneously in one patient in whom the measured diameter of the aortic valve ring was $23 \mathrm{~mm}$. The balloon catheter was then exchanged for the end hole catheter and the difference in pressure between the left ventricle and the ascending aorta was remeasured during systole. If this was $>40 \mathrm{~mm} \mathrm{Hg}$, and a larger balloon with an inflated diameter less than or equal to the measured diameter of the aortic valve was available, the procedure was repeated with the larger balloon. Finally, aortography was repeated in the same projection as the initial aortogram. Haemostasis was achieved by digital pressure.

\section{Results}

\section{IMMEDIATE RESULTS}

The aortic valve was crossed retrogradely in all but one patient, who was the first in the series. He underwent surgical valvotomy. In the remaining 33 patients, the mean peak to peak systolic pressure difference between the left ventricle and ascending aorta was 71 (30) $\mathrm{mm} \mathrm{Hg}$ (mean (SD)) before and 28 (19) $\mathrm{mm} \mathrm{Hg}(p<0.001)$ immediately after balloon dilatation (fig 1). In 13 patients we used sequential inflation of two balloons; the second was larger than the first. Four other patients had persisting gradients of $>40 \mathrm{~mm} \mathrm{Hg}$ after the use of a single balloon but the next balloon size available was considered too large to be used safely. In the only patient with heart failure the systolic pressure difference between the left ventricle and ascending aorta was reduced from 46 to $12 \mathrm{~mm} \mathrm{Hg}$, and this was associated with reductions in pulmonary artery systolic pressure and right atrial mean pressure from 70 to 48 and from 20 to $10 \mathrm{~mm} \mathrm{Hg}$ respectively. There was no relation between age and the extent of gradient relief.

\section{REPEAT CATHETERISATION}

To date 24 patients have had repeat cardiac catheterisation 2-19, mean 9 (4) months after the initial procedure. These repeat studies were performed under general anaesthesia in eight patients and basal sedation in the remainder. The mean peak to peak 
188

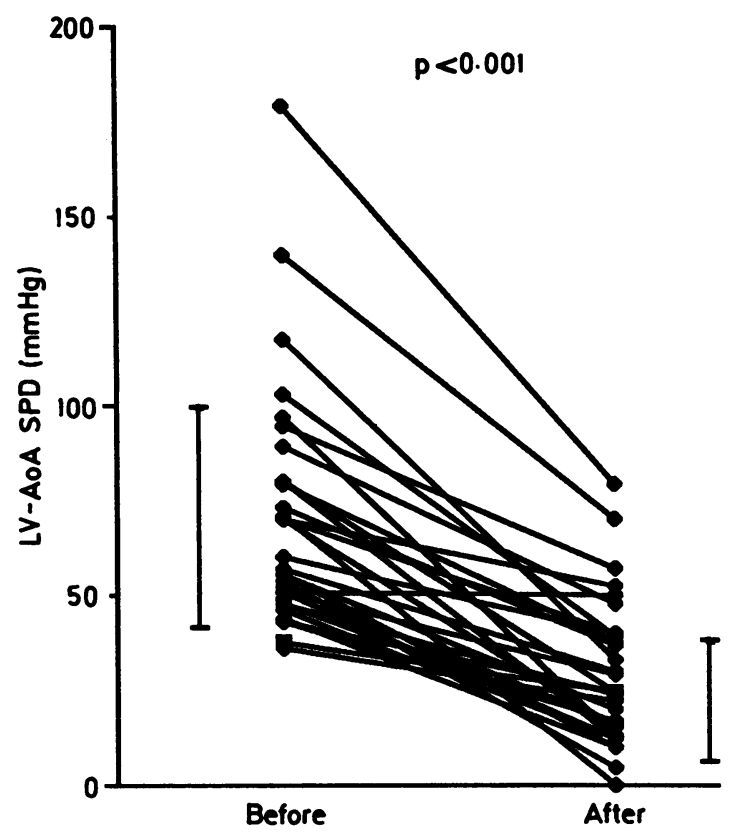

Fig 1 Systolic pressure difference between the left ventricle and ascending aorta before and immediately after balloon dilatation of the aortic valve. AoA, ascending aorta; $L V$, left ventricle; SPD, systolic pressure difference.

systolic pressure difference between the left ventricle and ascending aorta at restudy was $35(20) \mathrm{mm} \mathrm{Hg}$, compared with $31(20) \mathrm{mm} \mathrm{Hg}$ immediately after balloon dilatation (fig 2). It is notable that the two patients with the highest residual gradient immediately after balloon dilatation had spontaneous reduction in gradient at repeat catheterisation (from 70 to 40 and from 79 to $30 \mathrm{~mm} \mathrm{Hg}$ respectively). Repeat catheterisation was performed nine months after the initial procedure in each of these patients. The only patient who had a large increase in the systolic pressure difference between the left ventricle and ascending aorta at restudy was the patient who had twice undergone surgical valvotomy-via the transventricular approach as a neonate and by open valvotomy on cardiopulmonary bypass at one year. There had been a large residual transvalvar gradient after each operation. He has subsequently undergone successful aortic root replacement. The systolic pressure difference between the left ventricle and ascending aorta had increased from 12 to $35 \mathrm{~mm} \mathrm{Hg}$ in the patient who initially had heart failure and pulmonary hypertension. However, he has had major symptomatic improvement associated with a reduction in heart size on chest $x$ ray and an improvement in the left ventricular shortening fraction on echocardiography.
Two patients with residual gradients of 50 and:$59 \mathrm{~mm} \mathrm{Hg}$ had repeat balloon dilatation 14 and $\overrightarrow{2}$ : months after the initial procedure. We used a larges balloon than that used at the initial procedure in the first patient and two balloons inflated simultaneouslyes in the second. In both cases the residual gradient waso reduced (fig 2). There were only two other patients, with residual systolic pressure differences betweerf the left ventricle and ascending aorta of $>50 \mathrm{~mm} \overrightarrow{\mathrm{P}}$ $\mathrm{Hg}$. One, with a residual gradient of $65 \mathrm{~mm} \mathrm{Hg}$, is scheduled for repeat balloon dilatation. The other ${ }_{D}^{\omega}$ with a residual gradient of $53 \mathrm{~mm} \mathbf{~ H g}$, had a sub-? valvar component to her left ventricular outflow tracts obstruction and dilatation was not repeated.

COMPLICATIONS

ì

All patients tolerated the procedure well. Aortic regurgitation was apparent before balloon dilatationin nine $(27 \%)$ patients and not present in $24(73 \%){ }_{\infty}^{\top}$ After balloon dilatation (repeat balloon dilatation in two patients), aortic regurgitation had been induced in nine $(27 \%)$ patients-grade $I$ in eight $(24 \%)$ and grade II in one (3\%). Aortic regurgitation waso

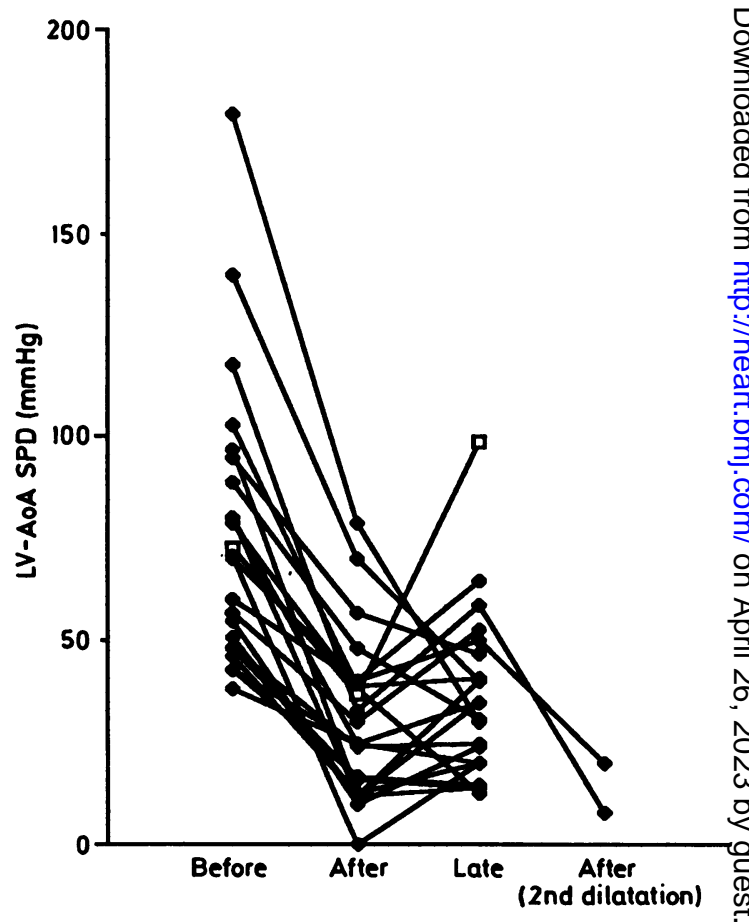

Fig 2 Systolic pressure difference between the left ventricle $T$ and ascending aorta before and after balloon dilatation of the $\stackrel{\vec{P}}{\vec{P}}$

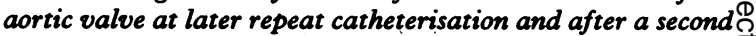
dilatation in two patients. The open symbol denotes the child $\vec{\nabla}$ with previous surgical valvotomy (see text). See fig 1 for abbreviations. 


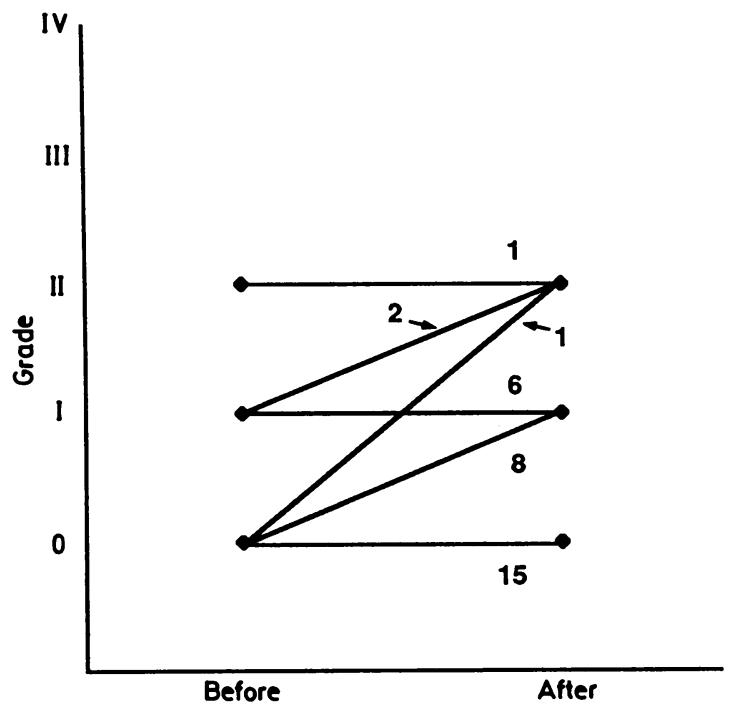

Fig 3 Angiographic grade of aortic regurgitation before and after balloon dilatation of the aortic valve. Numbers of patients are shown.

exacerbated, from grade I to II, in two (6\%) patients. In most (22 patients, $67 \%$ ) aortic regurgitation was not induced or was of similar severity before and after balloon dilatation (fig 3 ).

There was one major local arterial complication, when the external iliac artery was avulsed during withdrawal of a $15 \mathrm{~mm}$, 9 French gauge balloon catheter in a child weighing $9.5 \mathrm{~kg}$. This probably occurred because the 3.5 French gauge catheter introduced via the contralateral femoral artery to monitor pressure in the ascending aorta became entangled with the balloon catheter during withdrawal and was pulled down into the common iliac artery. This child underwent iliofemoral arterial reconstruction with an excellent (short term) result to date. This complication is avoidable, and our current practice is to withdraw the pressure monitoring catheter to the ipsilateral iliac artery before withdrawal of the balloon catheter. Two other patients required streptokinase when the leg pulses disappeared after the procedure. This was successful in one. In the other, pulses in the affected limb were palpable after streptokinase, but of reduced volume compared with the unaffected leg. No other local arterial complications were identified. Leg pulses remained palpable at the time of repeat catheterisation in all patients restudied to date.

\section{FOLLOW UP}

All patients remain well at follow up (time last seen) ranging from 1 to 24 , mean (SD) 13 (7) months. The patient with heart failure and pulmonary hyperten- sion before balloon dilatation is now symptom free at eight months follow up. A 14 year old boy with presyncope and dyspnoea is now symptom free after reduction of the systolic pressure difference between the left ventricle and ascending aorta of from 118 to $8 \mathrm{~mm} \mathrm{Hg}$. Syncope has not recurred in a 12 year old boy who had had unsuccessful surgical valvotomy. However, follow up is short (two months) and any benefit conferred by balloon dilatation in this case is doubtful (systolic pressure difference between the left ventricle and ascending aorta was reduced from 36 to $22 \mathrm{~mm} \mathrm{Hg}$ ). Several other children, with no reported symptoms initially, now have "more energy". No child has had a worsening of symptoms since balloon dilatation.

\section{Discussion}

Balloon dilatation of the aortic valve significantly reduced the transvalvar systolic pressure gradient both immediately after dilatation and at medium term repeat catheterisation in an unselected consecutive series of children with congenital aortic valve stenosis. The procedure was well tolerated, and was not difficult technically, in contrast with the technical difficulties experienced with very small infants. ${ }^{8}$ The child in whom the aortic valve could not be crossed at catheterisation was the first patient in the series, and he is the only child to have had surgical aortic valvotomy in either hospital during the study.

\section{COMPLICATIONS}

Aortic regurgitation was induced or exacerbated in $11(33 \%)$ patients. Our estimates of the severity of aortic regurgitation are fairly crude, but only one patient had worsening of the angiographic severity by more than one grade; the systolic pressure difference between the left ventricle and ascending aorta was concomitantly reduced from 97 to $13 \mathrm{~mm}$ Hg. Selection of balloon size is important in minimising the severity of aortic regurgitation after the procedure. In an experimental study in lambs, use of balloons with an inflated diameter of $120-150 \%$ of the aortic root diameter resulted in tears in the aortic valve and tears or haemorrhage or both in the anterior mitral valve leaflet, ventricular septum, and ascending aortic wall. ${ }^{9}$ Oversized balloons used intraoperatively in infants caused similar damage. ${ }^{10}$

Local arterial complications were less common than those encountered after balloon dilatation of the aortic valve in infants, or after balloon dilatation for recoarctation of the aorta. Our only major local complication, which required iliofemoral arterial reconstructive surgery, was potentially avoidable. Although none of the children in this series lost leg pulses, in contrast with experience in infants, ${ }^{8}$ the use 
of such large arterial catheters causes concern about the long term effect on limb growth. Introducing two balloons means slightly smaller catheters can be used, but both femoral arteries then become "at risk"."

\section{AORTIC VALVE AREA AND PRESSURE GRADIENTS}

In theory any improvement could be confirmed either by estimating the aortic valve area or the pressure gradients. We did not attempt to measure the aortic valve area. An increase in aortic valve area after balloon dilatation has been calculated by others, ${ }^{9}$ using the original Gorlin formula for aortic valve area, ${ }^{12}$ but in practice there are numerous problems in deriving accurate values. Firstly, the Gorlin formula ${ }^{12}$ has never been validated in children. Secondly, it is difficult to measure cardiac output accurately. ${ }^{1314}$ Thirdly, even if accurate values for mean transvalvar gradient and cardiac index are available, it may not be valid to use the Gorlin formula to compare changes in valve area before and after balloon dilatation. The original formula ${ }^{12}$ produces an apparent increase in aortic valve area for the same valve as cardiac output increases. Finally, when aortic regurgitation is present, a measurement of effective systemic cardiac output will underestimate the transvalvar aortic flow. If aortic regurgitation of different severity is present in the situations in which aortic valve area is to be compared, comparison of the values obtained will be further confused.

Transvalvar aortic systolic pressure gradients, on the other hand, are more easily measured than aortic valve area, although pressure in the ascending aortic rather than more peripheral systemic arterial pressure should be compared with left ventricular pressure. ${ }^{13}$ Systolic pressure differences between left ventricle and ascending aorta are more familiar to physicians, and a peak gradient of $>60 \mathrm{~mm} \mathrm{Hg}$ is advocated as a criterion for surgical valvotomy in congenital aortic stenosis. ${ }^{15}$ In the series of Hossack et al, ${ }^{1}$ assessment of the severity of aortic stenosis correlated better with the systolic pressure difference between the left ventricle and aorta than with calculated aortic valve area index, suggesting that the latter derived value did not provide additional information on the severity of disease in these patients.

Children in our study had all catheter gradients measured either under general anaesthesia or basal sedation. All but the child with heart failure had echocardiographic left ventricular shortening fraction within the normal range before and after balloon dilatation. While a transient reduction in cardiac output immediately after balloon dilatation might have contributed to a transient decrease in the systolic pressure differences between the left ventricle and ascending aorta, the persistence of this reduction at follow up catheterisation suggests $a_{c}$ genuine improvement in the degree of obstruction.

COMPARISON WITH VALVOTOMY

Aortic valvotomy in childhood is a palliative pro- $\mathbb{D}$ cedure. Although initial operative mortality is low, and survival at 15 years may be as good as $80-87 \%,{ }^{1-3} \mathrm{C}$ freedom from reoperation or a serious residual lesion. $\vec{\circ}$ over this period is as low as $14 \%{ }^{2}$ or $18 \%,{ }^{1}$ even in the $\vec{\omega}$ most experienced surgical centres. Consequently, an $\omega^{\circ}$ alternative form of palliation which might delay, or occasionally avoid, the need for operation, while $\sigma$ preserving the operative field, merits consideration. $\vec{i}$ Our initial experience with balloon dilatation of the $\vec{\infty}$ aortic valve suggests that the need for operation may be further delayed by repeat balloon dilatation in 의 some patients.

The importance of aortic regurgitation induced or ${ }_{\mathbb{D}}^{T}$ exacerbated by balloon dilatation is uncertain. After $\frac{\mathbb{O}}{2}$ open valvotomy aortic regurgitation was an indication for surgery in $30-36 \%$ of patients who required $₹$ reoperation..$^{1-3}$ Thus provided that an oversized bal- $\vec{\varnothing}$ loon is not used, the degree of aortic regurgitation induced by balloon aortic valvotomy may not be more than that induced by open surgical valvotomy.

With uncertainties about the natural history of $\frac{\partial}{\circ}$ congenital aortic stenosis in the era since the introduction of surgical intervention, automatic referral $\frac{\AA}{\varnothing}$ for open valvotomy of patients with congenital aortic $\underset{F}{\Rightarrow}$ stenosis and a peak systolic pressure gradient of $\frac{\circ}{3}$ $>60 \mathrm{~mm} \mathrm{Hg}$ between the left ventricle and ascending aorta ${ }^{15}$ is unappealing because it only achieves palliation. Some patients with such gradients will survive for many years without evident progression of the severity of their disease. ${ }^{1}$ There were only two? sudden deaths among 174 unoperated patients out of $\frac{\sigma}{3}$ a total series of 218 patients with congenital aortic stenosis followed for a mean period of nearly nine years. ${ }^{1}$ Both patients had been lost to follow up for nine and four years when they died suddenly. $\frac{}{2}$ However, it can be reasonably assumed that decom- $\frac{D}{0}$ pression of the left ventricle will have long term benefits for myocardial performance and balloon $N$ dilatation of the aortic valve may provide a low risk $N$ less traumatic alternative to open valvotomy. This initial form of palliation is suitable for many patients ${ }^{\omega}$ who are symptom free. Consequently, our criteria for balloon aortic valvotomy were more liberal than for ${ }^{\circ}$ open valvotomy. We do not suggest that there is an absolute indication for intervention in every patient + fulfilling these criteria. Our experience with balloon ${ }^{\circ}$ aortic valvotomy after unsuccessful open valvotomy $\underset{\mathbb{D}}{ }$ is limited to two patients, but suggests that balloon $\frac{\rho}{\Phi}$ valvotomy may be ineffective in such patients. 


\section{COMPARISON WITH OTHER STUDIES}

We obtained broadly the same reduction in gradient and low rate of serious complications as other centres. ${ }^{16-18}$ This accumulating experience suggests that the technique may be safely applied as a successful initial palliation over a wide age range in most children with congenital aortic stenosis.

\section{CONCLUSION}

Balloon dilatation of the aortic valve in a consecutive unselected series of children with congenital aortic stenosis provided reasonable relief of obstruction without inducing severe aortic regurgitation. Other complications have been infrequent. The technique has already had a major impact on our clinical practice. The encouraging short and medium term results indicate continuation of this policy of balloon dilatation for clinically significant aortic stenosis in children, but longer follow up is essential.

\section{References}

1 Hossack KF, Neutze JM, Lowe JB, Barratt-Boyes BG. Congenital valvar aortic stenosis. Natural history and assessment for operation. Br Heart J 1980;43:561-73.

2 Presbitero P, Somerville J, Revel-Chion R, Ross D. Open aortic valvotomy for congenital aortic stenosis. Late results. Br Heart $J$ 1982;47:26-34.

3 Hsieh K-S, Keane JF, Nadas AS, Bernhard WF, Castaneda AR. Long-term follow-up of valvotomy before 1968 for congenital aortic stenosis. Am J Cardiol 1986;58:338-41.

4 Sullivan ID, Robinson PJ, Macartney FJ, et al. Percutaneous balloon valvuloplasty for pulmonary valve stenosis in infants and children. Br Heart J 1985;54: 435-41.

5 Lababidi Z, Wu J-R, Walls JT. Percutaneous balloon aortic valvuloplasty: results in 23 patients. $\mathrm{Am} \mathrm{J}$ Cardiol 1984;53:194-7.

6 Rickards AF, Somerville J. Successful balloon aortic valvotomy in a child with a pulmonary hypertensive duct and aortic valve stenosis. Br Heart J 1986;56: 185-6.

7 Cribier A, Savin T, Saoudi N, Rocha P, Berland J, Letac B. Percutaneous transluminal valvuloplasty of acquired aortic stenosis in elderly patients: an alternative to valve replacement? Lancet 1986;i:63-7.

8 Wren C, Sullivan I, Bull C, Deanfield J. Percutaneous balloon dilatation of aortic valve stenosis in neonates and infants. Br Heart $J$ 1987;58:608-12.

9 Helgason H, Keane JF, Fellows KE, Kulik TJ, Lock JE. Balloon dilatation of the aortic valve: studies in normal lambs and in children with aortic stenosis. $J$ Am Coll Cardiol 1987;9:816-22.

10 Phillips RR, Gerlis LM, Wilson N, Walker DR. Aortic valve damage caused by operative balloon dilatation of critical aortic valve stenosis. Br Heart J 1987;57: 168-70.

11 Beekman RH, Rocchini AP, Crowley DC, et al. Aortic balloon valvuloplasty: two balloons are better than one [Abstract]. Circulation 1987;76(suppl IV):266.

12 Gorlin R, Gorlin SG. Hydraulic formula for calculation of the area of the stenotic mitral valve, other cardiac valves and central circulation shunts. Am Heart J 1951;41:1-29.

13 Carabello EA. Advances in the hemodynamic assessment of stenotic cardiac valves. J Am Coll Cardiol 1987;10:912-9.

14 Gorlin R. Calculations of cardiac valve stenosis: restoring an old concept for advanced applications. $\mathrm{J} \mathrm{Am}$ Coll Cardiol 1987;10:920-2.

15 Somerville J. Aortic stenosis and incompetence. In: Anderson RH, Macartney FJ, Shinebourne EA, Tynan M, eds. Paediatric cardiology. London: Churchill Livingstone, 1987:977-99.

16 Choy M, Beekman RH, Rocchini AP, et al. Percutaneous balloon valvuloplasty for valvar aortic stenosis in infants and children. Am J Cardiol 1987;59:1010-3.

17 Vogel M, Benson LN, Freedom RM, Rowe RD. Balloon valvuloplasty for isolated aortic stenosis in children-short-term results [Abstract]. J Am Coll Cardiol 1987;9:130A.

18 Sholler GF, Keane JF, Perry SB, Sanders SP, Lock JE. Balloon dilatation of aortic stenosis: influences of valve morphology and technique on outcome [Abstract]. Circulation 1987;76(suppl IV):554. 